\title{
Episodic evolution of prolactin receptor gene in mammals: coevolution with its ligand
}

\author{
Ying $\mathrm{Li}^{1,2,3}$, Michael Wallis ${ }^{4}$ and Ya-ping Zhang ${ }^{1,2}$ \\ ${ }^{1}$ Laboratory of Cellular and Molecular Evolution, and Molecular Biology of Domestic Animals, Kunming Institute of Zoology, Chinese Academy of Sciences, Kunming 650223, \\ People's Republic of China \\ ${ }^{2}$ Laboratory for Conservation and Utilization of Bio-resources, Yunnan University, Kunming 650091, People's Republic of China \\ ${ }^{3}$ The Graduate School, Chinese Academy of Sciences, Beijing 100039, People's Republic of China \\ ${ }^{4}$ Biochemistry Department, School of Life Sciences, University of Sussex, Brighton BNI 9QG, UK
}

(Requests for offprints should be addressed to Ya-ping Zhang, Laboratory of Cellular and Molecular Evolution, Kunming Institute of Zoology, Chinese Academy of Sciences, Kunming 650223, People's Republic of China; Email: zhangyp@ public.km.yn.cn)

\begin{abstract}
Divergence of proteins in signaling pathways requires ligand and receptor coevolution to maintain or improve binding affinity and/or specificity. In this paper we show a clear case of coevolution between the prolactin (PRL) gene and its receptor (prolactin receptor, PRLR) in mammals. First we observed episodic evolution of the extracellular and intracellular domains of the PRLR, which is closely consistent with that seen in PRL. Correlated evolution was demonstrated both between PRL and its receptor and between the two domains of the PRLR using Pearson's correlation coefficient. On comparing the ratio of the nonsynonymous substitution rate to synonymous substitution rate $\left(\omega=d_{N} / d_{S}\right)$ for each branch of the star phylogeny of mammalian PRLRs, separately for the extracellular domain (ECD) and the transmembrane domain/intracellular domain (TMD/ICD), we observed a lower $\omega$ ratio for ECD than TMD/ICD along those branches leading to pig, dog and rabbit but a higher ratio for ECD than TMD/ICD on the branches leading to primates, rodents and ruminants, on which bursts of rapid evolution were observed. These observations can be best explained by coevolution between PRL and its receptor and between the two domains of the PRLR.
\end{abstract}

Journal of Molecular Endocrinology (2005) 35, 411-419

\section{Introduction}

The prolactin (PRL) receptor (PRLR) is a single-pass transmembrane receptor belonging to the cytokine receptor superfamily (Kelly et al. 1991, Bole-Feysot et al. 1998). Receptors in this superfamily share a number of conserved motifs in both the extracellular domain (ECD) and the intracellular domain (ICD; Kelly et al. 1991, Goffin \& Kelly 1996), such as two pairs of disulfide-bonded cysteines and the WSXWS motif (Rozakis-Adcock \& Kelly 1991, 1992) in the ECD. Most sequence similarities are found in the ECD (Bole-Feysot et al. 1998), which is the binding domain for the ligand. In the PRLR there are 210 residues in the ECD and 24 residues in the transmembrane domain (TMD) in rat and human (Kelly et al. 1991). In some mammalian species there are several isoforms of the PRLR, but the long form is generally best characterized, and is the only isoform considered here.

The protein hormone PRL is involved in multiple biological processes in mammals (Bole-Feysot et al. 1998). It shows an episodic pattern of evolution in mammals: during much of mammalian evolution PRL evolved very slowly, but this near-stasis was interrupted by bursts of rapid change during the evolution of primates, artiodactyls, rodents and elephants (Wallis 2000). PRL has to bind to its receptor to fulfil its function. Accordingly, genes encoding PRL and PRLR should be subject to a mutual selection pressure to maintain or enhance affinity and/or specificity. This raises questions as to whether the PRLR gene in mammals shows a pattern of episodic evolution similar to that of its ligand and, if so, does this represent coevolution of hormone and receptor?

To answer these questions, we calculated evolutionary rates for branches of phylogenetic trees of all known mammalian PRLR genes. An episodic evolutionary pattern was evident and this pattern was independent of tree topology and divergence times used. The coincidence of rapid evolutionary phases with those demonstrated for the PRL gene, together with high Pearson's correlation coefficients and evidence from maximumlikelihood analysis of selective constraint variation suggest that the episodic evolution seen reflects coevolution of the PRLR and its ligand.

\section{Materials and methods}

\section{Sequences used}

PRLR gene cDNA sequences from 10 eutherian mammalian species are available in GenBank (accession 
Table 1 Sequences from GenBank used in this study

Accession no.

\begin{tabular}{|c|c|c|}
\hline & & \\
\hline & PRLR & PRL \\
\hline Species & & \\
\hline Human (Homo sapiens) & NM_000949 & V00566 \\
\hline Chimpanzee (Pan troglodytes) & This study (Fig. 1) & AADA01247695 and AADA01256522 \\
\hline Marmoset (Callithrix jacchus) & AJ272217 & AJ786353 \\
\hline Capuchin (Cebus apella) & AY227708 & Saki (Pithecia pithecia): AY851385-AY851388 \\
\hline Pig (Sus scrofa) & NM 001001868 & X14068 \\
\hline Cow (Bos taurus) & L02549 & J00022 \\
\hline Sheep (Ovis aries) & AF041257 & X13483 \\
\hline Red deer (Cervus elaphus) & X94953 & Elk (Cervus elaphus): AY373035 \\
\hline Dog (Canis familiaris) & This study (Fig. 1) & AY741405 \\
\hline Rabbit (Oryctolagus cuniculus) & J04510 & U27199 \\
\hline Rat (Rattus norvegicus) & M57668 & V01249 \\
\hline Mouse (Mus musculus) & NM_011169 & X02892 \\
\hline Possum (Trichosurus vulpecula) & AF098296 & AF054634 \\
\hline Chick (Gallus gallus) & NM_204854 & J04614 \\
\hline
\end{tabular}

numbers are shown in Table 1). In addition, genomic sequence information from dog and chimpanzee is available and therefore we can extract the PRLR coding region sequences of these two species (http:// genome.cse.ucsc.edu/cgi-bin/hgBlat). We used PRLR from a marsupial (brushtailed possum) and a bird (chicken) as outgroups in this study. In the chicken
PRLR gene, the cytokine receptor homology (CRH) domain is duplicated and forms two antenna-like structures, each of which corresponds to the ECD of mammalian PRLRs (Tanaka et al. 1992). To allow comparison with PRLR from mammalian species, we deleted the sequence corresponding to the second antenna-like structure. The PRL cDNA sequences from
10 20 30 40 50 60 70 80 90 100 110

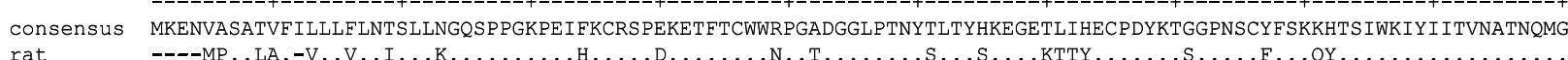
rat mouse capuchin marmoset human

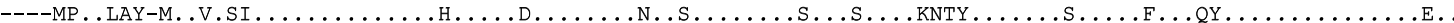

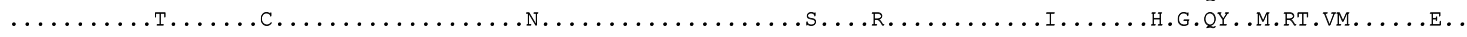

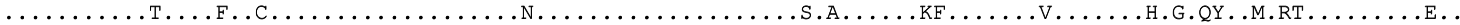

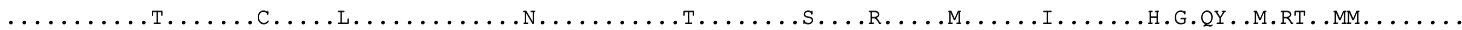

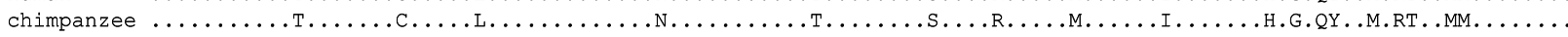

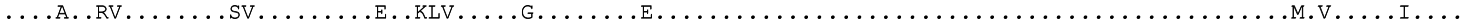

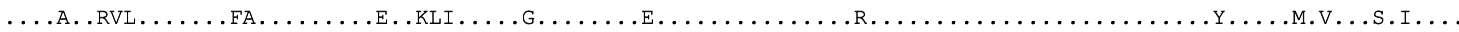

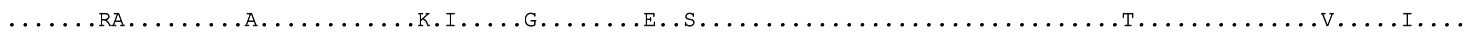

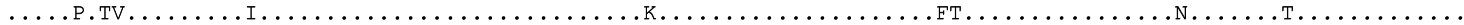

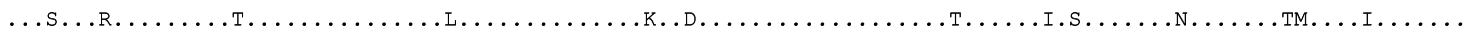

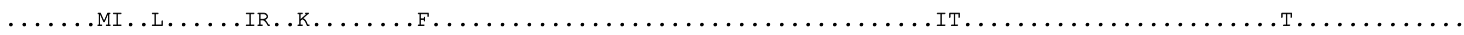

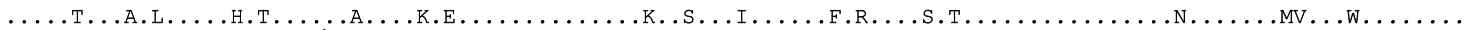

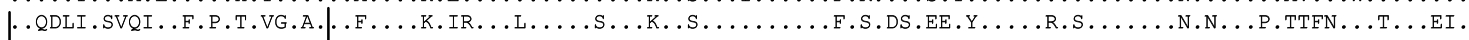
$\longleftrightarrow$ SP $\longrightarrow<$ ECD

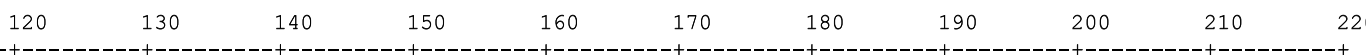

consensus SSSSDPLYVDVTYIVEPDPPVNLTLEVKQPEDRKPYLWIKWSPPTLTDVKSGWFTLQYEIRLKPEKAAEWETHFAGQQTQFKILSLYPGQKYLVQVRCKPDHGYWSEWSP

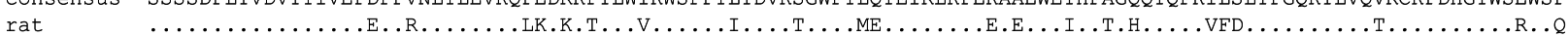

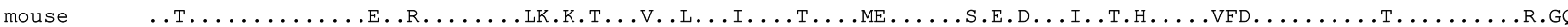

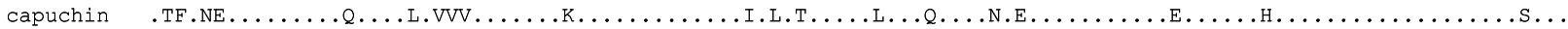

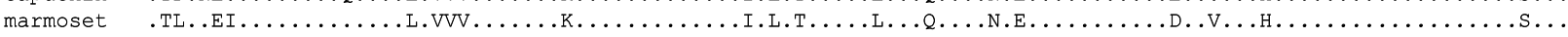
human chimpanzee cow

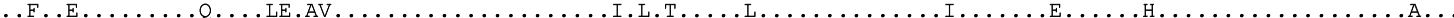

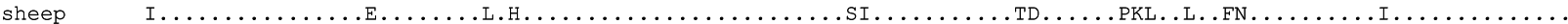

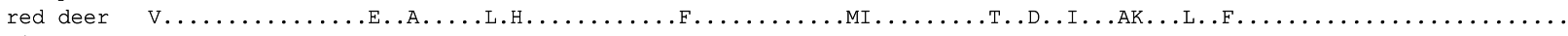

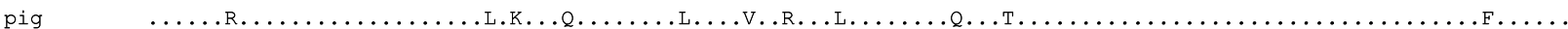
dog rabbit

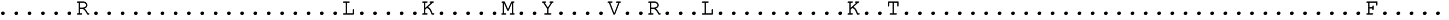

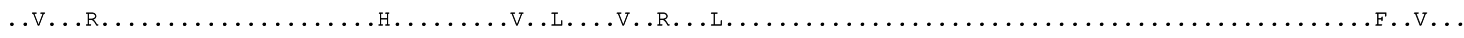

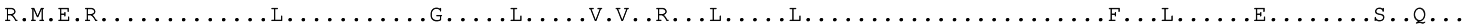
chick 
consensus

rat

mouse

capuchin

marmoset

human

chimpanzee

cow

sheep

red deer

pig

dog

rabbit

possum

chick

consensus

rat

mouse

capuchin

marmoset

human

chimpanzee

cow

sheep

red deer

pig

dog

rabbit

possum

chick

ESSIQI PNDFTMKDTTVWIFVAVLSAVICLIMVWAVALKGYSMVTCILPPVPGPKIKGFDTHLLEKGKSEELLSALGCQDFPPTSDCEDLLVEFLEVDDSEDQQLMPVHS

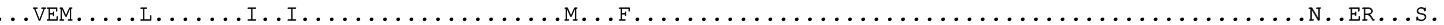

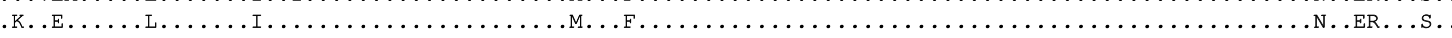

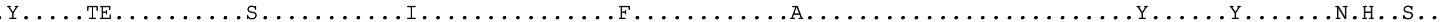

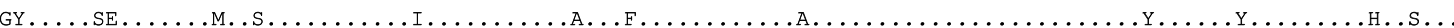

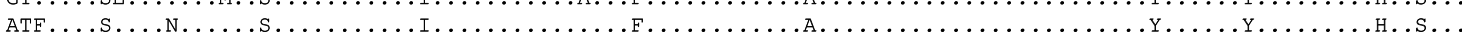

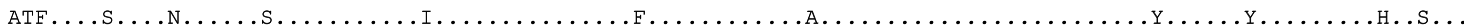

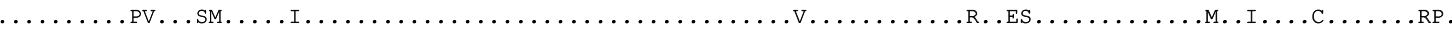

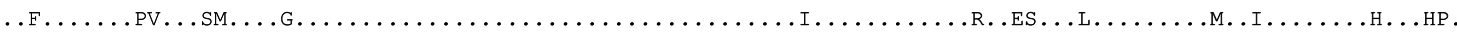

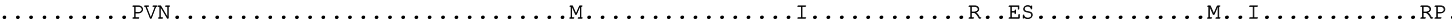

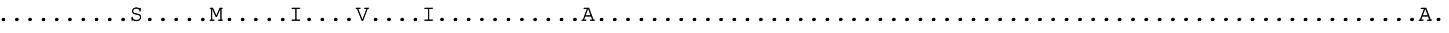

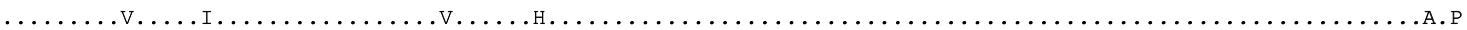

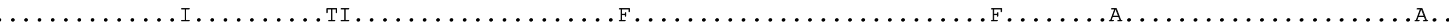

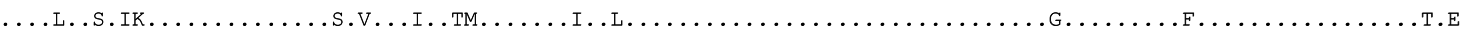

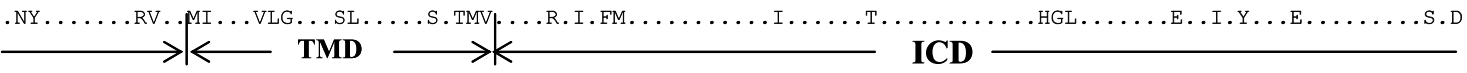

$\begin{array}{llllllllll}340 & 350 & 360 & 370 & 380 & 390 & 400 & 410 & 420 & 430\end{array}$

KEHPS QGMKPTHLD PDS DSGRGS CDS PSLLSEKCEEPQANP STF HTPEVI EKPENPETNHT- -WD PQS TSMEGK IPY FHA GGS KSS TWP LPQP-S QHN PRS SYHNIADVC

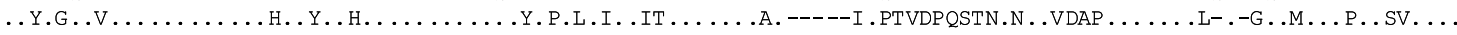

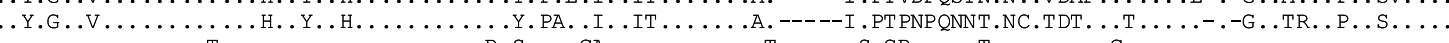

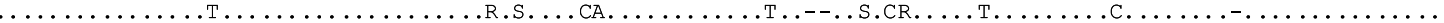

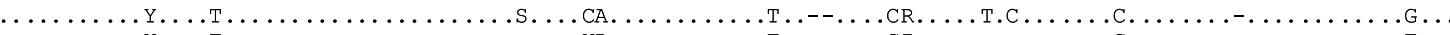

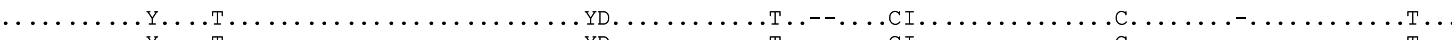

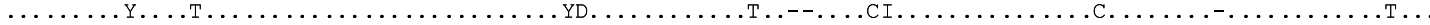

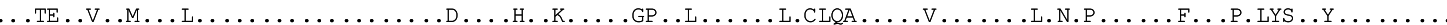

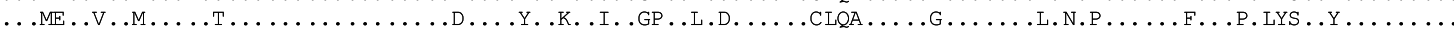

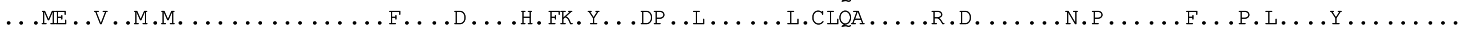

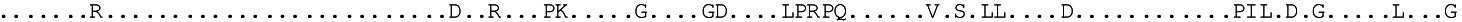

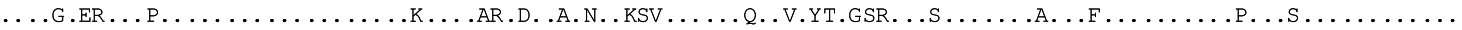

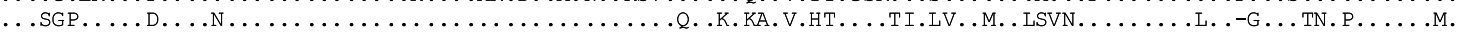

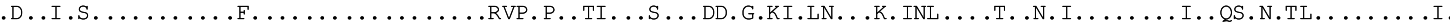
NG ...KNA.I.RKET ............R.TC.L.PVLQ.Q..RDVQ.KKAAKRS--.ET.YVAS.R. ALLSNSESA .....AV. LPNSQP.MFA..S.V.AH

ICD

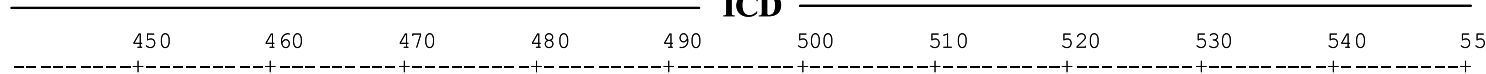

consensus

ELALGPAGA-- PATLLDKADKHALKS SKT IET GGE GKAAKQREVESF HSKTDQDTPWL---LPQEKTP FGS AKPLDYVEI HKVN-KDGALSLLPKQKENSGQPE KPGTPE

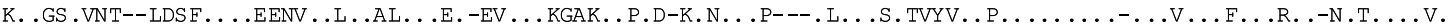

capuchin

marmoset

human

chimpanzee

sheep

red deer

pig

dog

rabbit

possum

chick

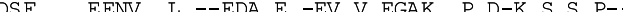

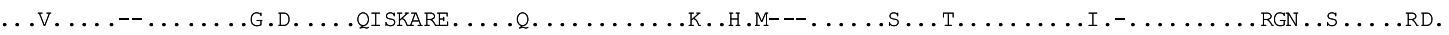

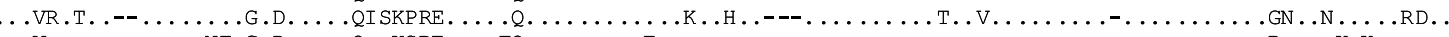

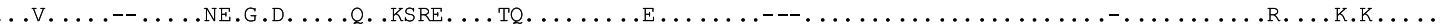

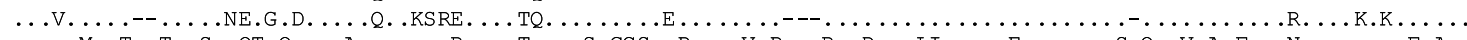

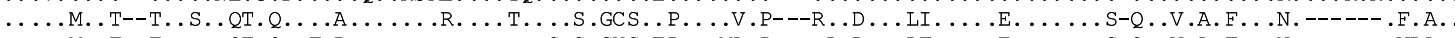

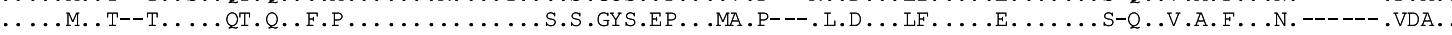

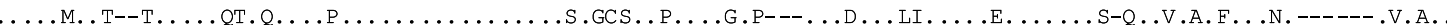

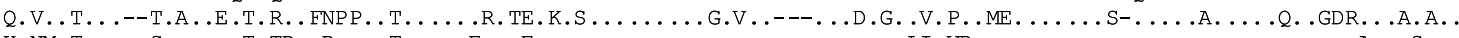

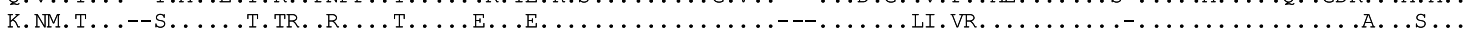

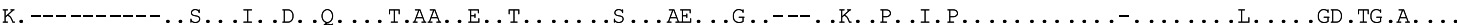

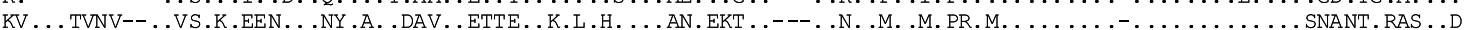
KIT.NTTNTNVA.V.VEDEEE.QSQC.L.ETIP..--ME. . G.M.NL . . .E. T.AQVKQNRSN.RL. .LD.ALM. . .V . . IRQ.EEPAV.L. H. . . KI. .YTISG

$\longrightarrow$ ICD

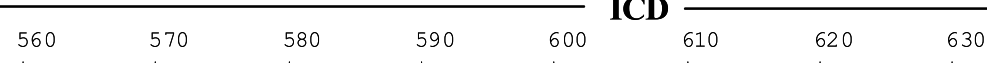

consensus TSKEYAKVSRVMDNNILVLVPDPRAQNVALFEESAKEA PPS LEQNQAEKDLAS FTA TPSNCRLQLGGLDYLDPACFMHSFH

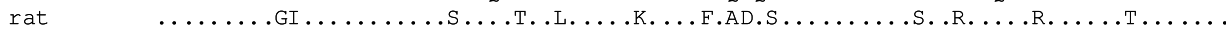

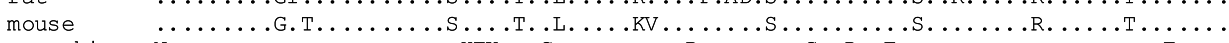

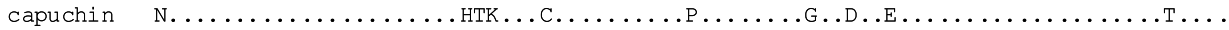

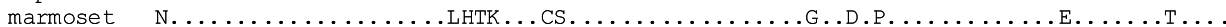

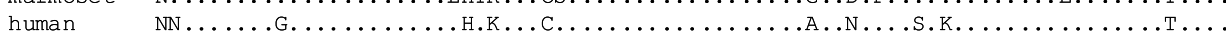

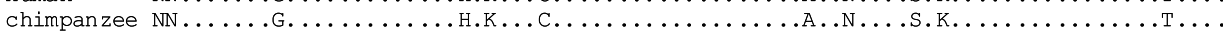

Cow A.........T.S..........LT.L..P..K...A.P----

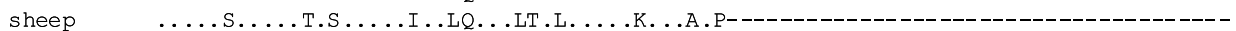

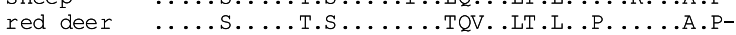

pig $\quad \ldots \ldots$..................PT.T..RP..P.A...G.TA.GH..HP..........Q

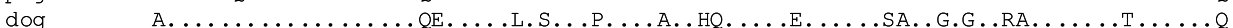

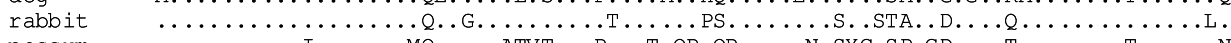

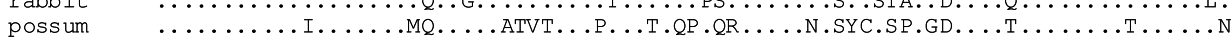

chick A...T............S.VPHTPASQ.P..TSQ..Q.G.V.NMSYCLTA..D.KRET..SE.M.SS..P.K

Figure 1 Alignment of inferred amino acid sequences of PRLR genes in mammals and a bird. The top line represents the consensus sequence, which was obtained by calculating the majority consensus at each each site using the DNAstar 5.0 software package (DNAstar, Madison, WI, USA). SP, signal peptide region. 
the same or closely related species as those available for PRLR were also obtained from GenBank (see Table 1).

\section{Phylogeny and divergence times}

For simplicity and convenience in comparing with PRLs, we used a star phylogeny for mammalian orders, which has been used to calculate evolutionary rates for PRL genes (Wallis 2000). This approximation assumes that the main orders of eutherian mammals diverged about 75 millon years before present (myBP) (Dayhoff 1972). Divergence times of other lineages were also kept consistent as far as possible with those used previously (Wallis 1994, 2000). They are: pig and ruminant artiodactyls, 55 myBP (Novacek 1982); red deer and cow/sheep, 27.8 myBP (Hassanin \& Douzery 2003); cow and sheep/ goat 17 myBP (Novacek 1982); mouse and rat, 25 myBP (O'huigin \& Li 1992); Old World monkey and hominoids, 36 myBP (Martin 1993); New World monkey and Old World monkey, 55 myBP (Martin 1993); capuchin monkey/marmoset, 22 myBP (Goodman et al. 1998).

\section{Evolutionary analyses}

All available cDNA sequences of mammalian PRL and PRLR genes were aligned (separately) using CLUSTALW (Thompson et al. 1994). Amino acid p-distance for each branch of the PRLR gene phylogeny was estimated, with deduced amino acid sequences as an input file, using the codeml program in the PAML package (Yang 1997). To test possible inconsistent rates of amino acid substitution for PRLR, the method of Li \& Bousquet (1992) implemented in RRTree (RobinsonRechavi \& Huchon 2000) was used for relative rate test. Pearson's correlation coefficient values were calculated by using the statistics software SPSS 10·0 1 (SPSS 1999). Maximum-likelihood analyses of substitution rates and changes in selective pressure were performed, using codeml in PAML (Yang 1997). We used the one-ratio model and the free-ratio model of the 'branch-specific' model to test for possible variation of selective pressure during evolution of PRLR gene along different mammalian lineages. The significance of the difference between the two models was estimated by calculating $2 \delta l$ and compared with the $\chi^{2}$ distribution, with degrees of freedom equal to the difference between the number of the parameters of the two models.

\section{Results and discussion}

\section{Evolutionary rates of PRLR gene in different mammalian lineages}

Aligned amino acid sequences of the PRLR gene from 14 species are shown in Fig. 1. Amino acid $p$-distances based on those sequences and the star phylogeny of eutherian mammals were estimated by codeml in PAML, with brushtailed possum and chicken PRLR being used as outgroups. PRLR is a transmembrane receptor containing a signal region, and we therefore divided the sequence data into four subsets representing four functional regions (signal peptide, ECD, TMD and ICD) when calculating amino acid substitution rates. Those rates were mapped onto the star phylogeny shown in Fig. 2.

For the ECD (Fig. 2B), the branches leading to pig, rabbit and $\operatorname{dog}$ PRLR evolved at rather low rates $\left(0.58 \times 10^{-9}, 0.59 \times 10^{-9}\right.$ and $0.86 \times 10^{-9}$ amino acid substitutions/site per year respectively). During the evolution of ruminants (designated branch Ae), rodents (branch Be) and primates (branch $\mathrm{Ce}$ ), the rates increased markedly to $5.04 \times 10^{-9}, 4.74 \times 10^{-9}$ and $8.57 \times 10^{-9}$ amino acid substitutions/site per year respectively, increases of 8-14-fold (for branches Ae, Be and Ce) relative to pig PRLR. An episodic evolutionary pattern has been noted for mammalian PRL, which in most of mammals evolved very slowly, but during the evolution of primates, rodents, ruminants and elephant showed substantial increases in evolutionary rate (Wallis 2000). Elephant PRLR is not available in GenBank, but for the other three lineages the bursts of rapid evolution in PRL correspond to the high evolutionary rates in the ECD of the receptor shown here. Thus this episodic evolutionary pattern observed in the ECD of the PRLR in mammals, and the difference in evolutionary rates between pig, rabbit, dog and those of primates, ruminants and rodents, probably result from coevolution with the ligand.

For the signal peptide and the TMD region (Fig. 2A and $\mathrm{C}$ ), we observed no episodic evolution that correlated with that shown by PRL, indicating that the correlation with PRL is specific to the domain involved in ligand binding, and further supporting the coevolution of hormone and receptor. On the other hand, the ICD (Fig. 2D), which is not directly involved in ligand binding, also shows episodic evolution similar to that of the ECD, though much less marked (Fig. 2D).

It is noteworthy that these episodes of rapid evolution occurred over a short period during the evolution of artiodactyls, rodents and primates (branches Ae, Be and Ce for ECD in Fig. 2B and branches Ai, Bi and $\mathrm{Ci}$ for ICD in Fig. 2D). In primates the rapid evolution occurred before the split of Old World monkeys and New World monkeys (branches Ce and Ci, Fig. 2), in artiodactyls it happened after pigs diverged from ruminants but before the divergence of red deer from cow and sheep (branches Ae and Ai, Fig. 2), and in rodents it occurred before the divergence of mouse and rat (branches Be and Bi, Fig. 2). In each of these three lineages the rate apparently fell back toward the 

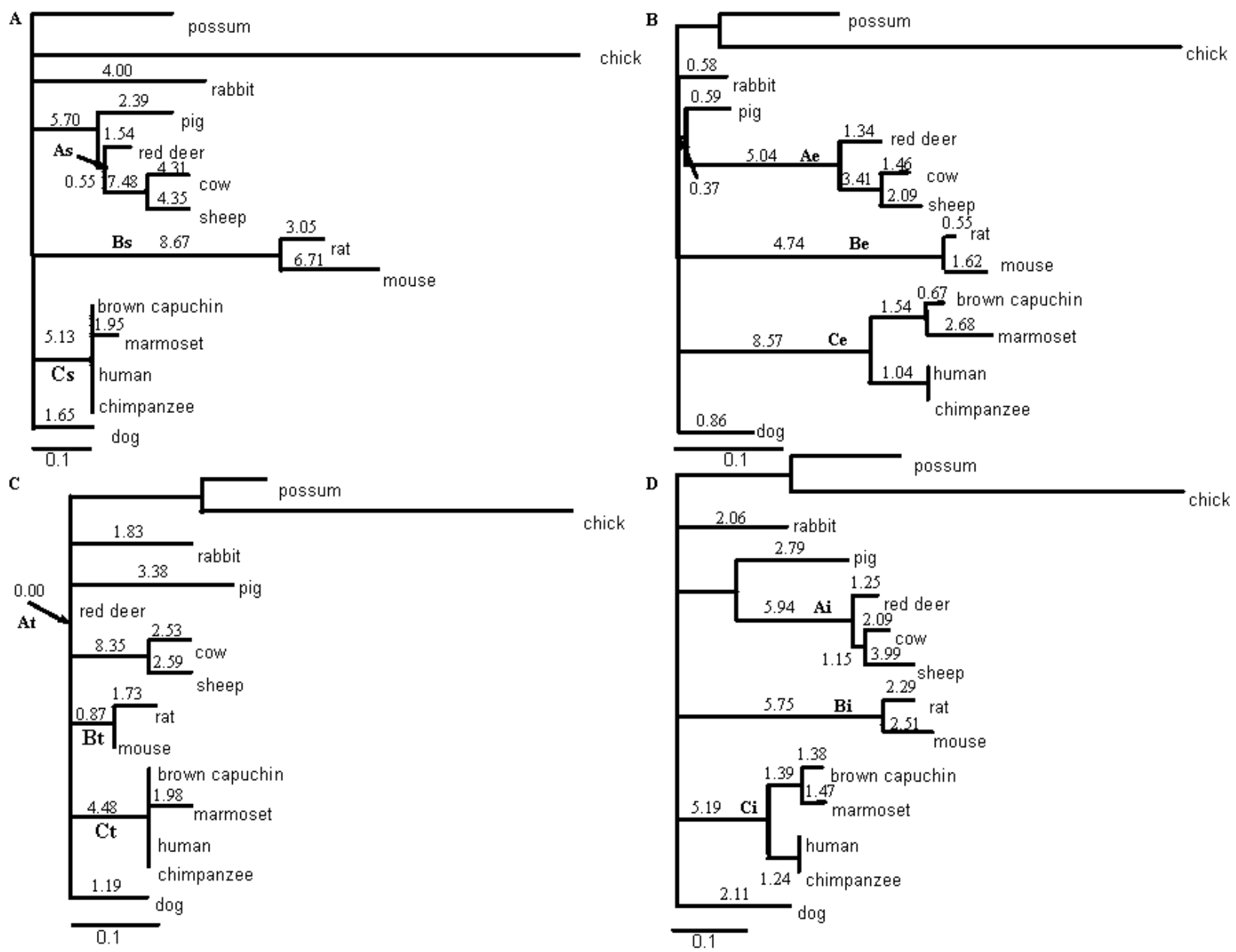

Figure 2 Star phylogeny of 12 eutherian mammalian species. The amino acid substitution rate ( $\times 10^{-9}$ substitutions/site per year) for each branch separately for signal region, ECD, TMD and ICD ( $A, B, C$ and $D$ respectively) is marked along the branches of the tree. Branches showing markedly higher evolutionary rates in ECD were marked as $\mathrm{Ae}, \mathrm{Be}$ and $\mathrm{Ce}$; the corresponding branches for the signal region were marked as As, Bs and Cs; those for TMD were marked as At, Bt and Ct; those for ICD were marked as $\mathrm{Ai}, \mathrm{Bi}$ and $\mathrm{Ci}$.

rate seen for rabbit, dog and pig after the phase of rapid change. These observations are consistent with the situation of PRL in mammals (Wallis 2000), further supporting the idea of coevolution between PRL and its receptor.

To eliminate possible bias due to use of erroneous phylogeny and divergence times, we recalculated evolutionary rates separately for the four domains of mammalian PRLR using an alternative phylogeny based on that of Kumar \& Hedges (1998) and alternative divergence times: 40 myBP for divergence of the Old and New World monkeys (Goodman et al. 1998) and 41 myBP as the divergence time for mouse and rat (Kumar \& Hedges 1998). The absolute values of the evolutionary rates changed, but the bursts of rapid change seen in the evolution of ECD and ICD of the PRLR in primates, rodents and artiodactyls, and the relatively low rates for rabbit, dog and pig, were preserved.

The significance of the rate variation observed in mammalian PRLR was tested using the relative rate test implemented in RRTree (Robinson-Rechavi \& Huchon 2000) with all sequences shown in Fig. 1 as input files, separately for signal region, ECD, TMD and ICD, and with chicken and brushtailed possum PRLR as outgroups. This method is independent of tree topology and divergence times. The test indicated that for the ECD region rate variations among pig, dog and rabbit were not statistically significant $(P>0 \cdot 05)$, but the rates on branches leading to ruminants, primates and rodents were significantly different from those of any for pig, $\operatorname{dog}$ and rabbit $(P<0 \cdot 01)$. For the signal region, TMD and ICD we observed no such tendency. 
Table 2 Correlation coefficients and related statistics

\begin{tabular}{|c|c|c|}
\hline \multirow[b]{2}{*}{ Pair } & $r$ value & \multirow[t]{2}{*}{$P$ value } \\
\hline & & \\
\hline PRL and PRLR & 0.820 & $<0.001$ \\
\hline Control & 0.054 & 0.67 \\
\hline PRL and ECD of PRLR & 0.770 & $<0.001$ \\
\hline Control & 0.031 & 0.80 \\
\hline PRL and TMD/ICD of PRLR & 0.780 & $<0.001$ \\
\hline Control & 0.010 & 0.94 \\
\hline ECD and TMD/ICD & 0.790 & $<0.001$ \\
\hline Control & 0.074 & 0.55 \\
\hline
\end{tabular}

' $r$ ' represents Pearson's correlation coefficient value and $P$ represents the significance of this value. Controls were chosen at random from either one of the comparison pair and were not believed to interact between the pairs of genes.

This confirms the observed episodic evolution in mammalian PRLR.

\section{Correlated evolution test}

Amino acid sequences of PRLR were paired with those of PRL from the same species or a very closely related species where an exact match was unavailable. We substituted PRLR from capuchin with saki and red deer with elk (Table 1). Pairwise amino acid distance correlations between ligand and receptor were calculated based on Pearson's correlation coefficient value $r$ (Press et al. 1988). The significance of this value was evaluated by two-tailed $t$-test, which gives the probability of getting the observed results ( $P$ value). Correlation coefficient values and the corresponding $P$ values are shown in Table 2. The correlation coefficient $r$ was 0.82 $(P<0 \cdot 01)$ when comparing pairwise distances between PRL and PRLR from the 12 eutherian species (full-length sequences), indicating a highly correlated coevolution between PRL and its receptor. Moreover, we also calculated the $r$ values for ligand versus ECD and ligand versus ICD. The $r$ value between ECD and PRL was $0.76(P<0.01)$, indicating highly correlated coevolution between PRL and the ligand-binding domain of PRLR. Interestingly, the ICD and the ligand also showed high correlation $(r=0 \cdot 79, P<0 \cdot 01)$. Possibly the ECD and ICD have coevolved, as has been reported for the phosphoglycerate kinase (PGK) gene $(r=0.79$ and $P<0.01$ for the $\mathrm{N}$ - and C-terminal domains; Coh et al. 2000). To test this hypothesis, we calculated $r$ between ECD and ICD. A high correlation coefficient value $(r=0 \cdot 80, P<0 \cdot 01)$ confirmed our conjecture that the two domains of PRLR show correlated coevolution.

To evaluate the reliability of this method, we carried out a negative control for each test, in which the distance of one gene was randomly assigned to an incorrect partner. We then calculated the correlation coefficients based on these incorrect partners. The results showed no
Table 3 Maximum-likelihood $\omega$ ratios for the PRLR data

\begin{tabular}{|c|c|}
\hline$\omega$ for ECD & $\omega$ for $T M D / I C D$ \\
\hline 0.72 & 0.42 \\
\hline $0 \cdot 82$ & 0.46 \\
\hline $1 \cdot 51$ & 0.92 \\
\hline $0 \cdot 20$ & 0.38 \\
\hline $0 \cdot 14$ & 0.32 \\
\hline $0 \cdot 17$ & 0.32 \\
\hline
\end{tabular}

Rodent (branch a)

Primate (branch b)

Dog

Rabbit

Lineage
Rodent (branch a)
Primate (branch b)
Ruminant (branch c)
Dog
Pig
Rabbit

significant correlation between these random distances (Table 2).

\section{Variation of selective pressure for PRLR along different mammalian lineages}

The sequence similarity among cytokine receptors is mostly found in the ECD (Bole-Feysot et al. 1998), so we expected that the ECD should be subject to more strict selective constraint than other regions and that if the episodic evolution seen in PRLR is the result of coevolution with its ligand, the selective constraints variation pattern for the two genes should be consistent. To test this hypothesis, we used the branchspecific models of the maximum-likelihood method implemented in PAML (Yang 1997). The free-ratio model, which assumes an independent $\omega$ value (the ratio of the nonsynonymous substitution rate to synonymous substitution rate; $\omega=d_{\mathrm{N}} / d_{\mathrm{S}}$ ) for every branch, is significantly better than the one-ratio model $(2 \delta l=70 \cdot 18$, $P<0 \cdot 001, \mathrm{df}=21)$, suggesting great selective pressure variation among different lineages of mammalian PRLR. So we used the $\omega$ ratio for each branch separately for ECD and TMD/ICD domains under the free-ratio model (Table 3). We did observe stricter purifying selection in the ECD region than in the TMD/ICD region during the evolution of pig, rabbit and $\operatorname{dog}$ PRLR, which evolved relatively slowly. But along the branches showing the rapid evolutionary phases of primates, rodents and ruminants we find the opposite tendency (Table 3). Wallis (2000) reported a similar increase of $d_{\mathrm{N}} / d_{\mathrm{S}}$ values along those lineages showing sustained rapid evolution. Therefore, the correlation between high $d_{\mathrm{N}} / d_{\mathrm{S}}$ values of ligand and the ligand-binding domain of the receptor further supports the coevolution of these two genes. The increase of $d_{\mathrm{N}} / d_{\mathrm{S}}$ is also observed in the TMD/ICD regions for those branches showing rapid evolution, and this finding can be best explained by correlated evolution between the two domains of PRLR, which is consistent with our above correlation analysis. Furthermore, using a different method, the maximum-parsimony-based 
method proposed by Zhang et al. (1998), did not change our main results, suggesting that our result is independent of the method used.

\section{Biological significance of episodic evolution of PRLR and its ligand}

Our results provide clear evidence for episodic evolution of the PRLR gene in mammals, and of coevolution with the PRL gene. This study is based on the only 12 eutherian mammal species, representing five mammalian orders, for which the PRLR gene sequence is available and the analysis will have to be refined as PRLRs from more species are identified. Nevertheless, it seems clear that these results reflect adaptive changes, although the basis of these changes is not clear. The main function of PRL in mammals is the regulation of mammary growth and development and of lactation, and although there are substantial differences in mammary physiology and anatomy between different mammalian groups, these do not primarily involve the direct actions of PRL. The binding affinity of PRL to its receptor varies somewhat between species, and it is particularly noticeable that the affinity for the homologous receptor is often much lower than for the heterologous receptor (e.g. Bignon et al. 1994, Sandowski et al. 1995, Gertler et al. 1996). Thus, Bignon et al. (1994) showed that rabbit PRL was at least 100 times less effective than ovine PRL in competing for binding to the ECD of the rabbit PRLR, although for biological activity the difference was much less marked. However, it should be noted that PRL has a number of actions in non-mammary tissues in mammals and indeed is produced at many extrapituitary sites (Ben-Jonathan et al. 1996, Bole-Feysot et al. 1998). The relative importance of these various actions may differ between mammalian groups and could provide the basis for the observed episodic evolution, possibly via the mechanism of function-switching (Wallis 1997, Forsyth \& Wallis 2002).

Functional evolutionary changes involving the interaction between a ligand and its receptor may affect affinity and/or specificity. Although increased affinity might provide a basis for a more effective hormonereceptor interaction, it is unlikely that affinity is a limiting factor in determining the effectiveness of PRL in mammals, particularly since as discussed above the binding affinity between the homologous hormone and its receptor may be substantially less than can be achieved when the same receptor binds PRL from a different species. Changes in specificity could provide a more likely basis for the episodic evolution, especially if associated with the appearance of related hormones (see below) and/or changes in the relative importance of different actions of PRL mediated by different receptors. Although there is no evidence for more than one PRLR gene in any of the mammalian species considered here, there is clear evidence for different forms of the receptor protein, produced by different splicing patterns and/or post-translational modification. These processes give rise, for example, to forms of the receptor in which the ICD is truncated (Bole-Feysot et al. 1998), and it may be that such different forms of the receptor mediate different actions of the hormone. Detailed analysis of the changes that occur during the episodes of rapid coevolution of PRL and its receptor can potentially indicate whether such changes are likely to alter the specificity of hormone-receptor interaction, but in the absence of a three-dimensional structure for any homologous PRL-PRLR complex, such analysis would be difficult and over-speculative. Analysis of the changes that occur in PRL during the burst of evolution in primates in light of the three-dimensional structure of human PRL (Keeler et al. 2003) indicates that none of these occurs in binding site 1 (Wallis et al. 2005), but binding site 2 is not well-defined. This contrasts with the situation for primate growth hormone, where there are many substitutions in both sites 1 and 2 during the episode of rapid evolution (Liu et al. 2001, Wallis et al. 2001).

Particularly interesting is that for each of the lineages on which bursts of rapid evolution of PRLR were observed, a cluster of growth hormone- or PRL-like genes, including genes for placental lactogen, has been reported, rather than a single pituitary PRL gene. Thus, clusters of PRL-like genes are found in rodents and ruminants (Forsyth \& Wallis 2002) and a cluster of growth hormone-like genes is found in higher primates (Chen et al. 1989, Golos et al. 1993, Wallis et al. 2001, Wallis \& Wallis 2002). Most other mammalian groups, including pig, rabbit and dog, do not appear to have such a cluster (Talamantes et al. 1980, Forsyth \& Wallis 2002), and in the case of dog this was confirmed by a BLAST search of the genome sequence. Placental lactogens appear to use the PRLR signaling pathway (Golos et al. 1993, Soares et al. 1998, Herman et al. 2000, Biener et al. 2003) like pituitary PRL. This might suggest that the episodic evolution observed for the PRLR reflects adaptation to the presence of multiple ligands. However, in primates and ruminants at least this episode preceded the duplications that gave rise to placental lactogens (Wallis 1992, 1996, 2000). An explanation that has been put forward for the corresponding burst of rapid evolution seen in primate growth hormones is that a period of fluctuating demands on the hormone led to rapid evolution by the function-switching mechanism (Wallis 1997, Forsyth \& Wallis 2002) and that this was resolved by gene duplication so that the several actions that had been undertaken by growth hormone were divided among placental and pituitary hormones. A similar explanation may apply in the case of PRL. 
Coevolution between PRL and the ECD of its receptor is perhaps not unexpected, but the observation of coevolution between PRL and the receptor ICD is more surprising. However, the interaction between PRL and PRLR to give the receptor dimerization necessary for biological function is very weak when only the ECD of the receptor is studied (Bignon et al. 1994, Gertler et al. 1996) and may be stabilized or modified by interactions between the ICDs of the two receptor molecules. In this case the ICD would be contributing to hormonereceptor interactions, as well as mediating intracellular signaling, and coevolution with the ICD and PRL would be less surprising.

\section{Acknowledgements}

This work was supported by the National Natural Science Foundation of China $(30430110,30021004)$, the Natural Science Foundation of Yunnan Province and the Chinese Academy of Sciences. The authors declare that there is no conflict of interest that would prejudice the impartiality of this scientific work.

\section{References}

Ben-Jonathan N, Mershon JL, Allen DL \& Steinmetz RW 1996 Extrapituitary prolactin: Distribution, regulation, functions, and clinical aspects. Endocrine Reviewes 17 639-669.

Biener E, Martin C, Daniel N, Frank SJ, Centonze VE, Herman B, Djiane JJ \& Gertler A 2003 Ovine placental lactogen-induced heterodimerization of ovine growth hormone and prolactin receptors in living cells is demonstrated by fluorescence resonance energy transfer microscopy and leads to prolonged phosphorylation of signal transducer and activator of transcription (STAT)1 and STAT3. Endocrinology 144 3532-3540.

Bignon C, Sakal E, Belair L, Chapnik-Cohen N, Djiane J \& Gertler A 1994. Preparation of the extracellular domain of the rabbit prolactin receptor expressed in Escherichia coli and its interaction with lactogenic hormones. Fournal of Biological Chemistry 269 3318-3324.

Bole-Feysot C, Goffin V, Edery M, Binart N \& Kelly PA 1998 prolactin (PRL) and its receptor: actions, signal transduction pathways and phenotypes observed in PRL receptor knockout mice. Endocrine Reviews 19 225-268.

Chen, EY, Liao YC, Smith DH, Barrera-Saldana HA, Gelinas RE \& Seeburg PH 1989 The human growth hormone locus: nucleotide sequence, biology, and evolution. Genomics 4 479-497.

Coh CS, Bogan AA, Joachimiak M, Walther D \& Cohen FE 2000 Co-evolution of proteins with their interaction partners. Fournal of Molecular Biology 299 283-293.

Dayhoff MO 1972 Atlas of Protein Sequence and Structure, vol 5. Washington DC: National Biomedical Research Foundation.

Forsyth IA \& Wallis M 2002 Growth hormone and prolactin-molecular and functional evolution. Fournal of Mammary Gland Biology and Neoplasia 7 291-312.

Gertler A, Grosclaude J, Strasburger CJ, Nir S \& Djiane J 1996 Real-time kinetic measurements of the interactions between lactogenic hormones and prolactin-receptor extracellular domains from several species support the model of hormone-induced transient receptor dimerization. Fournal of Biological Chemistry 271 24482-24491.

Goffin V \& Kelly PA 1996 Prolactin and growth hormone receptors. Clinical Endocrinology 45 247-255.

Golos TG, Durning M, Fisher JM \& Fowler PD 1993 Cloning of four growth hormone/chorionic somatomammotropin-related complementary deoxyribonucleic acids differentially expressed during pregnancy in the rhesus monkey placenta. Endocrinology 133 $1744-1752$.

Goodman M, Porter CA, Czelusniak J, Page SL, Schneider H, Shoshani J, Gunnell G \& Groves CP 1998 Toward a phylogenetic classification of primates based on DNA evidence complemented by fossil evidence. Molecular Phylogenetics and Evolution 9 585-598.

Hassanin A \& Douzery EJP 2003 molecular and morphological phylogenies of ruminantia and the alternative position of the moschidae. Systematic Biology 52 206-228.

Herman A, Bignon C, Daniel N, Grosclaude J, Gertler A \& Djiane J 2000 Functional heterodimerization of prolactin and growth hormone receptors by ovine placental lactogen. Fournal of Biological Chemistry 275 6295-6301.

Keeler C, Dannies PS \& Hodsdon ME 2003 The tertiary structure and backbone dynamics of human prolactin. Fournal of Molecular Biology 328 1105-1121.

Kelly PA, Djiane J, Postel-Vinay MC \& Edery M 1991 The prolactin/growth hormone receptor family. Endocrinology Reviews 12 235-251.

Kumar S \& Hedges SB 1998 A molecular timescale for vertebrate evolution. Nature 392 917-920.

Li P \& Bousquet J 1992 Relative-rate test for nucleotide substitutions between two lineages. Molecular Biology and Evolution 9 1185-1189.

Liu J-C, Makova KD, Adkins, RM, Gibson S \& Li W-H 2001 Episodic evolution of growth hormone in primates and emergence of the species specificity of human growth hormone receptor. Molecular Biology and Evolution 18 945-953.

Martin RD 1993 Primate origins: plugging the gaps. Nature 363 223-234.

Novacek MJ 1982 Information for molecular studies from anatomical and fossil evidence on higher eutherian phylogeny. In Macromolecular Sequences in Systematic and Evolutionary Biology, pp 3-41. Ed M Goodman. New York: Plenum Press.

O'huigin C \& Li WH 1992 The molecular clock ticks regularly in muroid rodents and hamsters. Fournal of Molecular Evolution 35 377-384.

Press WH, Teukolsky SA, Vetterling WT \& Flannery BP 1988 Numerical Recipes in C: The Art of Scientific Computing. Cambridge: Cambridge University Press.

Robinson-Rechavi M \& Huchon D 2000 RRTree: relative-rate tests between groups of sequences on a phylogenetic tree. Bioinformatics 16 296-297.

Rozakis-Adcock M \& Kelly PA 1991 Mutational analysis of the ligand binding domain of the prolactin receptor. Fournal of Biological Chemistry 266 16472-16477.

Rozakis-Adcock M \& Kelly PA 1992 Identification of ligand binding determinants of the prolactin receptor. Fournal of Biological Chemistry $2677428-7433$.

Sandowski Y, Nagano M, Bignon C, Djiane J, Kelly PA \& Gertler A 1995 Preparation and characterization of recombinant prolactin receptor extracellular domain from rat. Molecular and Cellular Endocrinology 115 1-11.

Soares MJ, Miller H, Orwig KE, Peters TJ \& Dai G 1998 The uteroplacental prolactin family and pregnancy. Biology of Reproduction 58 273-284.

SPSS 1999 SPSS Base 10·0 for Windowes User's Guide. Chicago IL: SPSS. Talamantes F, Ogren L, Markoff E, Woodard S \& Madrid J 1980 Phylogenetic distribution, regulation of secretion, and prolactin-like effects of placenta lactogens Federation Proceedings 39 $2582-2587$. 
Tanaka M, Maeda K, Okubo T \& Nakashima K 1992 Double antenna structure of chicken prolactin receptor deduced from the cDNA sequence Biochemical and Biophysical Research Communications $188490-496$.

Thompson JD, Higgins DG \& Gibson TJ 1994 CLUSTAL W: improving the sensitivity of progressive multiple sequence alignment through sequence weighting, positions-specific gap penalties. Nucleic Acids Research 22 4673-4680.

Wallis M 1992 The expanding growth hormone/prolactin family. Journal of Molecular Endocrinology 9 185-188.

Wallis M 1994 Variable evolutionary rates in the molecular evolution of mammalian growth hormones. Fournal of Molecular Evolution 38 619-627.

Wallis M 1996 The molecular evolution of vertebrate growth hormones: a pattern of near-stasis interrupted by sustained bursts of rapid change. Fournal of Molecular Evolution $\mathbf{4 3}$ 93-100.

Wallis M 1997 Function switching as a basis for bursts of rapid change during the evolution of pituitary growth hormone. Fournal of Molecular Evolution 44 348-350.
Wallis M 2000 Episodic evolution of protein hormones: molecular evolution of pituitary prolactin. Fournal of Molecular Evolution $\mathbf{5 0}$ 465-473.

Wallis M \& Wallis OC 2002 Characterizing of the GH gene cluster in a new-world monkey, the marmoset (Callithrix jacchus). Fournal of Molecular Endocrinology 2 89-97.

Wallis OC, Zhang YP \& Wallis M 2001 Molecular evolution of growth hormone $(\mathrm{GH})$ in primates. Characterisation of the $\mathrm{GH}$ genes from slow loris and marmoset defines an episode of rapid evolutionary change. Fournal of Molecular Endocrinology 26 249-258.

Wallis OC, Mac-Kwashie AO, Makri G \& Wallis M 2005 Molecular evolution of prolactin in primates. Fournal of Molecular Evolution $60600-614$

Yang Z 1997 PAML: a program package for phylogenetic analysis by maximum likelihood. CABIOS 13 555-556.

Zhang J, Rosenberg HF \& Nei M 1998 Positive Darwinian selection after gene duplication in primate ribonuclease genes. PNAS 95 3708-3713.

Received on 12 August 2005

Accepted 12 August 2005 